\title{
2,2,3,3,4,4,5,5-Octafluorohexane-1,6-diol. Corrigendum
}

Kylie Feightner, ${ }^{a}$ Douglas R. Powell ${ }^{\mathrm{b}}$ and Christopher M. Burba ${ }^{\mathrm{a} *}$

${ }^{a}$ Department of Natural Sciences, Northeastern State University, 611 N. Grand Ave., Tahlequah, OK 74464, USA, and b Department of Chemistry and Biochemistry, University of Oklahoma, 101 Stephenson Parkway, Norman, OK 73019, USA. *Correspondence e-mail: burba@nsuok.edu

In the paper by Feightner et al. [IUCrData (2020), 5, x200445], there was an error in the name of the title compound.

The name of the title compound in the paper by Feightner et al. (2020) is incorrect and should be '2,2,4,4,5,5,7,7-octafluoro-3,6-dioxaoctane-1,8-diol'. The scheme and chemical data within the article are correct.

\section{References}

Feightner, K., Powell, D. R. \& Burba, C. M. (2020). IUCrData, 5, x200445. 


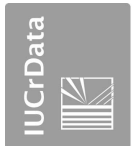

\section{IUCrData}

ISSN 2414-3146

Received 25 February 2020

Accepted 31 March 2020

Edited by A. J. Lough, University of Toronto, Canada

Keywords: crystal structure; fluorinated glycol; hydrogen bonding.

CCDC reference: 1993931

Structural data: full structural data are available from iucrdata.iucr.org

\section{2,2,3,3,4,4,5,5-Octafluorohexane-1,6-diol}

\author{
Kylie Feightner, ${ }^{\mathrm{a}}$ Douglas R. Powell ${ }^{\mathrm{b}}$ and Christopher M. Burba ${ }^{\mathrm{a} *}$
}

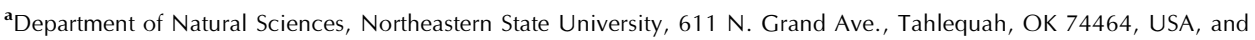
b Department of Chemistry and Biochemistry, University of Oklahoma, 101 Stephenson Parkway, Norman, OK 73019, USA. *Correspondence e-mail: burba@nsuok.edu

In the crystal of the title compound, $\mathrm{C}_{6} \mathrm{H}_{6} \mathrm{~F}_{8} \mathrm{O}_{4}, \mathrm{O}-\mathrm{H} \cdots \mathrm{O}$ hydrogen bonds involving the hydroxy groups connect the molecules, forming a two-dimensional network parallel to (100). These hydrogen-bonding interactions appear to drive the $\mathrm{O}-\mathrm{C}-\mathrm{C}-\mathrm{O}$ torsion angles into a gauche-trans-trans series of conformations along the backbone of the molecule.
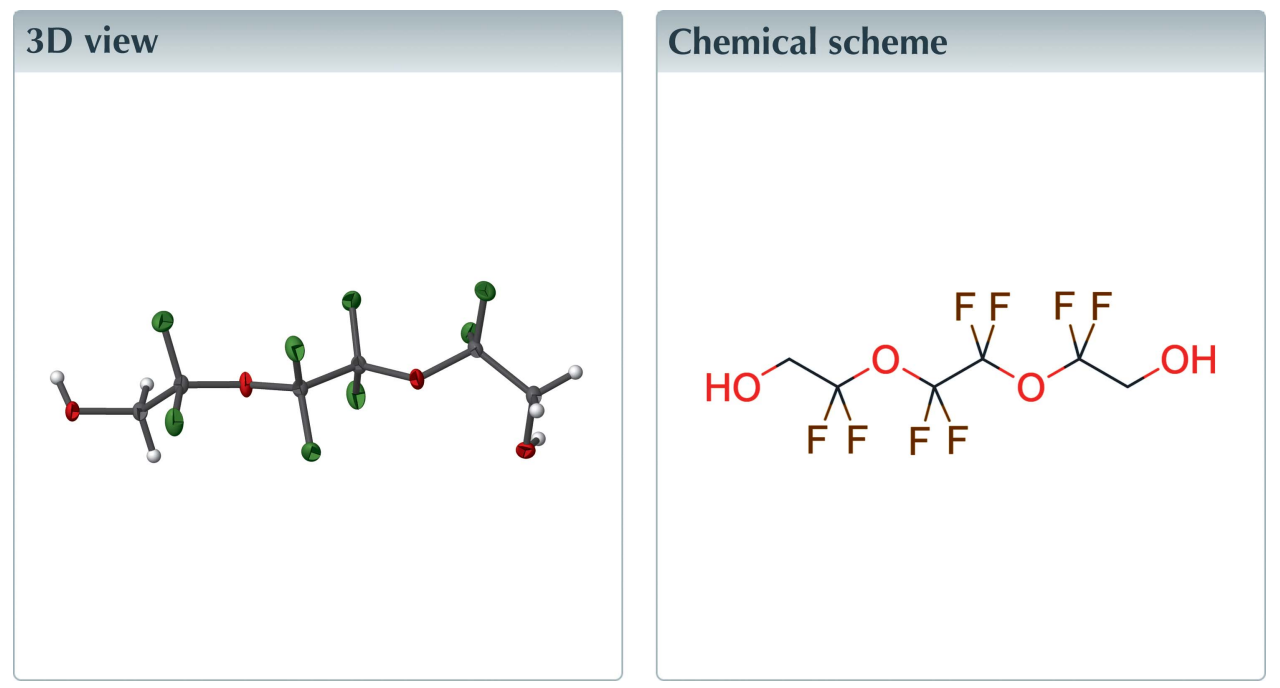

\section{Structure description}

Ionic liquids have attracted considerable interest as solvents for a variety of applications. 'Solvate' ionic liquids (SILs) are a new class of ionic liquids that consist of equimolar mixtures of inorganic salts and molecular solvents capable of chelating the cations of the salt (Ueno et al., 2012, 2015; Mandai et al., 2014, 2015). Most research on SILs focus on methyl-capped ethylene oxide molecular solvents, which are collectively known as 'glymes'. Structural variation of the chelating compound will undoubtedly influence cation-solvent interactions and provide alternative means for tuning SIL properties (Saito et al., 2016). Our lab has pursued this line of research by examining partially fluorinated molecular solvents for SIL applications. During our experiments, we isolated and determined the structure of the title compound, a partially fluorinated derivative of triethylene glycol. The molecular structure of the title compound is shown in Fig. 1. In the crystal, $\mathrm{O}-\mathrm{H} \cdots \mathrm{O}$ hydrogen bonds involving the terminal hydroxyl groups (see Table 1) connect the molecules, forming a two-dimensional network parallel to (100) (Fig. 2). In addition, a weak intermolecular $\mathrm{C}-\mathrm{H} \cdots \mathrm{F}$ hydrogen bond is observed within this network. These hydrogen-bonding interactions appear to drive the $\mathrm{O}-\mathrm{C}-\mathrm{C}-\mathrm{O}$ torsion angles into a gauche-trans-trans series of conformations along the backbone of the molecule: $\mathrm{O} 1-\mathrm{C} 1-\mathrm{C} 2-\mathrm{O} 2=66.3(2), \mathrm{O} 2-\mathrm{C} 3-\mathrm{C} 4-\mathrm{O} 2=-168.91(15)$, and $\mathrm{O} 3-$ $\mathrm{C} 5-\mathrm{C} 6-\mathrm{O} 4=-177.92(15)^{\circ}$. By way of comparison, the $\mathrm{O}-\mathrm{C}-\mathrm{C}-\mathrm{O}$ torsion angles 


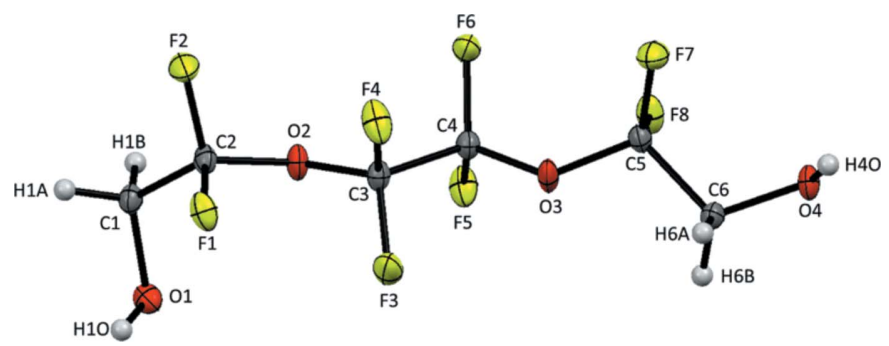

Figure 1

The molecular structure of the title compound. Displacement ellipsoids are shown at $50 \%$ probability level.

are gauche in monoglyme (Yoshihiro et al., 1996) and longer chain glymes (Johansson et al., 2010; Hyun et al., 2001; Tadokoro, 1964).

\section{Synthesis and crystallization}

2,2,3,3,4,4,5,5-Octafluoro-1,6-hexanediol (1.94 $\mathrm{mmol})$ was added to a 1:1 molar ratio mixture of lithium bis(trifluoromethanesulfonyl)imide $(2.3 \mathrm{mmol})$ and $2,2^{\prime}$-[ethane-1,2diylbis(oxy)]di(ethan-1-ol) (commonly known as triethylene glycol; $2.3 \mathrm{mmol}$ ). The resulting mixture was stirred at $353 \mathrm{~K}$ for $6 \mathrm{~h}$ to produce a homogenous, viscous solution. Colorless, plate-shaped single crystals formed from the solution upon standing over a period of days.

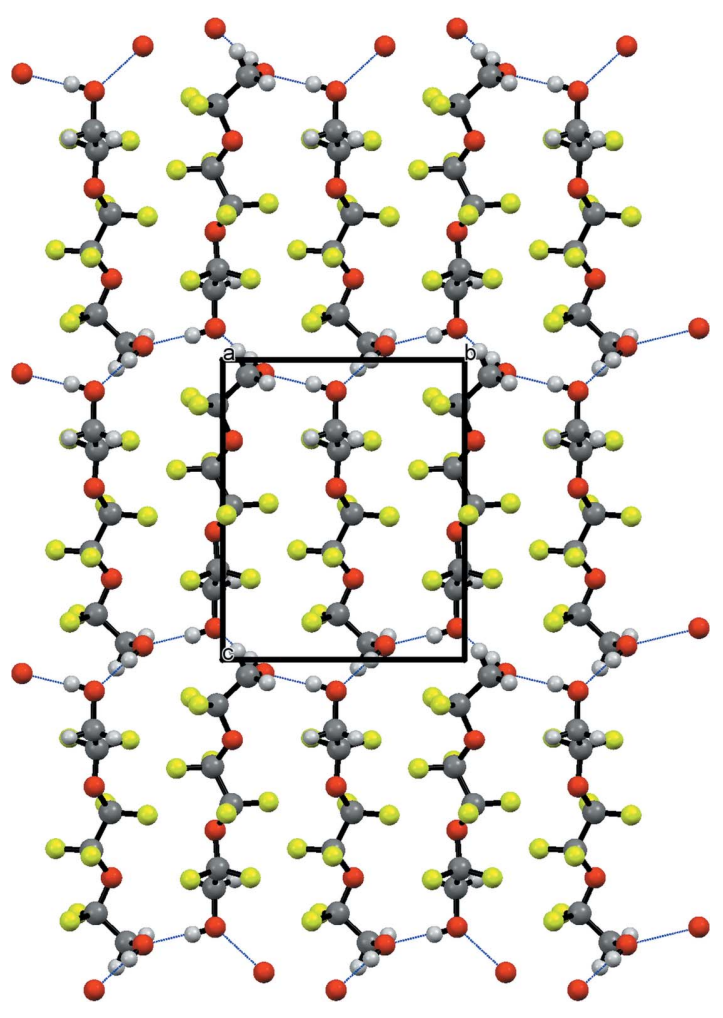

Figure 2

Part of the crystal structure with hydrogen bonds shown as dashed lines.
Table 1

Hydrogen-bond geometry $\left(\AA,{ }^{\circ}\right)$.

\begin{tabular}{lllll}
\hline$D-\mathrm{H} \cdots A$ & $D-\mathrm{H}$ & $\mathrm{H} \cdots A$ & $D \cdots A$ & $D-\mathrm{H} \cdots A$ \\
\hline $\mathrm{O} 1-\mathrm{H} 1 O \cdots \mathrm{O} 4^{\mathrm{i}}$ & $0.84(4)$ & $1.87(4)$ & $2.705(2)$ & $172(3)$ \\
$\mathrm{O} 4-\mathrm{H} 4 O \cdots 1^{\mathrm{ii}}$ & $0.85(4)$ & $1.82(4)$ & $2.661(2)$ & $173(3)$ \\
$\mathrm{C} 6-\mathrm{H} 6 B \cdots \mathrm{F} 4^{\mathrm{iii}}$ & 0.99 & 2.52 & $3.416(2)$ & 151 \\
\hline
\end{tabular}

Symmetry codes: (i) $x, y, z+1$; (ii) $-x+1, y-\frac{1}{2},-z+1$; (iii) $-x+1, y+\frac{1}{2},-z+1$.

Table 2

Experimental details.

\begin{tabular}{|c|c|}
\hline \multicolumn{2}{|l|}{ Crystal data } \\
\hline Chemical formula & $\mathrm{C}_{6} \mathrm{H}_{6} \mathrm{~F}_{8} \mathrm{O}_{4}$ \\
\hline$M_{\mathrm{r}}$ & 294.11 \\
\hline Crystal system, space group & Monoclinic, $P 2_{1}$ \\
\hline Temperature $(\mathrm{K})$ & 100 \\
\hline$a, b, c(\AA)$ & $\begin{array}{l}5.3009(8), 8.6250(12) \\
10.6976(14)\end{array}$ \\
\hline$\beta\left({ }^{\circ}\right)$ & $91.146(5)$ \\
\hline$V\left(\AA^{6}\right)$ & $489.00(12)$ \\
\hline$Z$ & 2 \\
\hline Radiation type & Мо $K \alpha$ \\
\hline$\mu\left(\mathrm{mm}^{-1}\right)$ & 0.25 \\
\hline Crystal size $(\mathrm{mm})$ & $0.49 \times 0.49 \times 0.05$ \\
\hline \multicolumn{2}{|l|}{ Data collection } \\
\hline Diffractometer & Bruker Photon II CMOS \\
\hline Absorption correction & $\begin{array}{l}\text { Multi-scan (SADABS; Krause et } \\
\quad \text { al., 2015) }\end{array}$ \\
\hline$T_{\min }, T_{\max }$ & $0.543,0.746$ \\
\hline $\begin{array}{l}\text { No. of measured, independent and } \\
\text { observed }[I>2 \sigma(I)] \text { reflections }\end{array}$ & $14748,2985,2896$ \\
\hline$R_{\text {int }}$ & 0.049 \\
\hline$(\sin \theta / \lambda)_{\max }\left(\AA^{-1}\right)$ & 0.715 \\
\hline \multicolumn{2}{|l|}{ Refinement } \\
\hline$R\left[F^{2}>2 \sigma\left(F^{2}\right)\right], w R\left(F^{2}\right), S$ & $0.038,0.100,1.00$ \\
\hline No. of reflections & 2985 \\
\hline No. of parameters & 170 \\
\hline No. of restraints & 1 \\
\hline $\mathrm{H}$-atom treatment & $\begin{array}{l}\mathrm{H} \text { atoms treated by a mixture of } \\
\text { independent and constrained } \\
\text { refinement }\end{array}$ \\
\hline$\Delta \rho_{\max }, \Delta \rho_{\min }\left(\mathrm{e} \AA^{-3}\right)$ & $0.65,-0.40$ \\
\hline
\end{tabular}

Computer programs: APEX3 (Bruker, 2018), SAINT (Bruker, 2016), SHELXT (Sheldrick, 2015a), SHELXL2018/3 (Sheldrick, 2015b) and Mercury (Macrae et al., 2020).

\section{Refinement}

Crystal data, data collection methods, and structural refinement details are provided in Table 2. The absolute structure of the title compound could not be established in the refinement reported here.

\section{Acknowledgements}

The authors wish to thank the Department of Natural Science, Northeastern State University for financial support of this project.

\section{Funding information}

Funding for this research was provided by: American Chemical Society Petroleum Research Fund (grant No. 57803- 
UR10); National Science Foundation (grant No. CHE1726630).

\section{References}

Bruker (2016). SAINT. Bruker Nano Inc., Madison, Wisconsin, USA. Bruker (2018). APEX3. Bruker Nano Inc., Madison, Wisconsin, USA.

Hyun, J.-K., Dong, H., Rhodes, C. P., Frech, R. \& Wheeler, R. A. (2001). J. Phys. Chem. B, 105, 3329-3337.

Johansson, P., Grondin, J. \& Lassègues, J. C. (2010). J. Phys. Chem. A, 114, 10700-10705.

Krause, L., Herbst-Irmer, R., Sheldrick, G. M. \& Stalke, D. (2015). J. Appl. Cryst. 48, 3-10.

Macrae, C. F., Sovago, I., Cottrell, S. J., Galek, P. T. A., McCabe, P., Pidcock, E., Platings, M., Shields, G. P., Stevens, J. S., Towler, M. \& Wood, P. A. (2020). J. Appl. Cryst. 53, 226-235.
Mandai, T., Yoshida, K., Tsuzuki, S., Nozawa, R., Masu, H., Ueno, K., Dokko, K. \& Watanabe, M. (2015). J. Phys. Chem. B, 119, 15231534.

Mandai, T., Yoshida, K., Ueno, K., Dokko, K. \& Watanabe, M. (2014). Phys. Chem. Chem. Phys. 16, 8761-8772.

Saito, S., Watanabe, H., Ueno, K., Mandai, T., Seki, S., Tsuzuki, S., Kameda, Y., Dokko, K., Watanabe, M. \& Umebayashi, Y. (2016). J. Phys. Chem. B, 120, 3378-3387.

Sheldrick, G. M. (2015a). Acta Cryst. A71, 3-8.

Sheldrick, G. M. (2015b). Acta Cryst. C71, 3-8.

Tadokoro, H., Chatani, Y., Yoshihara, T., Tahara, S. \& Murahashi, S. (1964). Makromol. Chem. 73, 109-127.

Ueno, K., Tatara, R., Tsuzuki, S., Saito, S., Doi, H., Yoshida, K., Mandai, T., Matsugami, M., Umebayashi, Y., Dokko, K. \& Watanabe, M. (2015). Phys. Chem. Chem. Phys. 17, 8248-8257.

Ueno, K., Yoshida, K., Tsuchiya, M., Tachikawa, N., Dokko, K. \& Watanabe, M. (2012). J. Phys. Chem. B, 116, 11323-11331.

Yoshihiro, Y., Hidehiro, U. \& Yuji, O. (1996). Chem. Lett. 25, 443-444. 


\section{full crystallographic data}

IUCrData (2020). 5, x200445 [https://doi.org/10.1107/S2414314620004459]

\section{2,2,3,3,4,4,5,5-Octafluorohexane-1,6-diol}

\section{Kylie Feightner, Douglas R. Powell and Christopher M. Burba}

\section{2,2,3,3,4,4,5,5-Octafluorohexane-1,6-diol}

\section{Crystal data}

$\mathrm{C}_{6} \mathrm{H}_{6} \mathrm{~F}_{8} \mathrm{O}_{4}$

$M_{r}=294.11$

Monoclinic, $P 2_{1}$

$a=5.3009(8) \AA$

$b=8.6250(12) \AA$

$c=10.6976(14) \AA$

$\beta=91.146(5)^{\circ}$

$V=489.00(12) \AA^{3}$

$Z=2$

\section{Data collection}

Bruker Photon II CMOS

diffractometer

Radiation source: microfocus sealed tube

$\omega$ and $\varphi$ scans

Absorption correction: multi-scan

(SADABS; Krause et al., 2015)

$T_{\min }=0.543, T_{\max }=0.746$

14748 measured reflections

Refinement

Refinement on $F^{2}$

Least-squares matrix: full

$R\left[F^{2}>2 \sigma\left(F^{2}\right)\right]=0.038$

$w R\left(F^{2}\right)=0.100$

$S=1.00$

2985 reflections

170 parameters

1 restraint

Primary atom site location: dual

Secondary atom site location: difference Fourier map
$F(000)=292$

$D_{\mathrm{x}}=1.997 \mathrm{Mg} \mathrm{m}^{-3}$

Mo $K \alpha$ radiation, $\lambda=0.71073 \AA$

Cell parameters from 9936 reflections

$\theta=3.0-30.5^{\circ}$

$\mu=0.25 \mathrm{~mm}^{-1}$

$T=100 \mathrm{~K}$

Plate, colourless

$0.49 \times 0.49 \times 0.05 \mathrm{~mm}$

2985 independent reflections 2896 reflections with $I>2 \sigma(I)$

$R_{\text {int }}=0.049$

$\theta_{\max }=30.5^{\circ}, \theta_{\min }=3.0^{\circ}$

$h=-7 \rightarrow 7$

$k=-12 \rightarrow 12$

$l=-15 \rightarrow 15$

Hydrogen site location: mixed

$\mathrm{H}$ atoms treated by a mixture of independent and constrained refinement

$w=1 /\left[\sigma^{2}\left(F_{\mathrm{o}}^{2}\right)+(0.075 P)^{2}+0.078 P\right]$

where $P=\left(F_{\mathrm{o}}^{2}+2 F_{\mathrm{c}}{ }^{2}\right) / 3$

$(\Delta / \sigma)_{\max }<0.001$

$\Delta \rho_{\max }=0.65 \mathrm{e} \AA^{-3}$

$\Delta \rho_{\min }=-0.40$ e $\AA^{-3}$

Absolute structure: Refined as an inversion twin

Absolute structure parameter: 0.5 (6)

\section{Special details}

Geometry. All esds (except the esd in the dihedral angle between two 1.s. planes) are estimated using the full covariance matrix. The cell esds are taken into account individually in the estimation of esds in distances, angles and torsion angles; correlations between esds in cell parameters are only used when they are defined by crystal symmetry. An approximate (isotropic) treatment of cell esds is used for estimating esds involving l.s. planes.

Refinement. Refined as a 2-component inversion twin. 
Fractional atomic coordinates and isotropic or equivalent isotropic displacement parameters $\left(\AA^{2}\right)$

\begin{tabular}{|c|c|c|c|c|}
\hline & $x$ & $y$ & $z$ & $U_{\text {iso }} * / U_{\text {eq }}$ \\
\hline F1 & $0.7033(3)$ & $0.36867(16)$ & $0.87486(11)$ & $0.0193(3)$ \\
\hline $\mathrm{F} 2$ & $1.1035(3)$ & 0.40107 (18) & $0.84955(14)$ & $0.0238(3)$ \\
\hline F3 & $0.4638(2)$ & $0.4558(2)$ & $0.65888(13)$ & $0.0241(3)$ \\
\hline F4 & $0.7907(3)$ & $0.31004(16)$ & $0.63351(13)$ & $0.0232(3)$ \\
\hline F5 & $0.7219(3)$ & $0.68928(15)$ & $0.52189(12)$ & $0.0214(3)$ \\
\hline F6 & $1.0050(2)$ & $0.51638(17)$ & $0.48334(12)$ & $0.0192(3)$ \\
\hline F7 & 0.7953 (2) & $0.36716(19)$ & $0.26323(13)$ & $0.0225(3)$ \\
\hline F8 & 0.7493 & $0.61560(17)$ & $0.26968(12)$ & $0.0204(3)$ \\
\hline O1 & $0.6422(3)$ & $0.67095(18)$ & $0.95429(14)$ & $0.0166(3)$ \\
\hline $\mathrm{H} 1 \mathrm{O}$ & $0.556(6)$ & $0.614(5)$ & $1.001(3)$ & $0.020 *$ \\
\hline $\mathrm{O} 2$ & $0.8372(3)$ & $0.54080(17)$ & $0.73056(13)$ & $0.0155(3)$ \\
\hline $\mathrm{O} 3$ & $0.5994(3)$ & $0.46959(19)$ & $0.42741(13)$ & $0.0167(3)$ \\
\hline $\mathrm{O} 4$ & $0.4017(3)$ & $0.46960(18)$ & 0.10482 (13) & $0.0155(3)$ \\
\hline $\mathrm{H} 4 \mathrm{O}$ & $0.395(6)$ & $0.376(5)$ & $0.081(3)$ & $0.019 *$ \\
\hline $\mathrm{C} 1$ & $0.8841(4)$ & $0.6040(2)$ & $0.94631(18)$ & $0.0155(3)$ \\
\hline H1A & 0.935916 & 0.560859 & 1.028644 & $0.019 *$ \\
\hline $\mathrm{H} 1 \mathrm{~B}$ & 1.007975 & 0.684471 & 0.923235 & $0.019^{*}$ \\
\hline $\mathrm{C} 2$ & 0.8810 & $0.4762(2)$ & $0.84895(17)$ & $0.0144(3)$ \\
\hline C3 & $0.7124(3)$ & $0.4577(2)$ & $0.63955(17)$ & $0.0137(3)$ \\
\hline $\mathrm{C} 4$ & $0.7627(3)$ & $0.5366(2)$ & $0.51257(17)$ & $0.0130(3)$ \\
\hline $\mathrm{C} 5$ & $0.6362(3)$ & $0.4807(2)$ & $0.29963(17)$ & $0.0133(3)$ \\
\hline C6 & 0.3799 (3) & $0.4651(2)$ & $0.23611(17)$ & $0.0138(3)$ \\
\hline H6A & 0.301564 & 0.365806 & 0.260933 & $0.017 *$ \\
\hline H6B & 0.269087 & 0.550509 & 0.263425 & $0.017 *$ \\
\hline
\end{tabular}

Atomic displacement parameters $\left(\AA^{2}\right)$

\begin{tabular}{lllllll}
\hline & $U^{11}$ & $U^{22}$ & $U^{33}$ & $U^{12}$ & $U^{13}$ & $U^{23}$ \\
\hline F1 & $0.0299(6)$ & $0.0142(5)$ & $0.0140(6)$ & $-0.0041(5)$ & $0.0021(5)$ & $0.0020(4)$ \\
F2 & $0.0236(6)$ & $0.0300(7)$ & $0.0180(6)$ & $0.0135(5)$ & $-0.0003(5)$ & $0.0004(5)$ \\
F3 & $0.0166(5)$ & $0.0397(8)$ & $0.0161(5)$ & $-0.0061(6)$ & $0.0016(4)$ & $0.0022(6)$ \\
F4 & $0.0426(8)$ & $0.0115(5)$ & $0.0154(6)$ & $0.0009(5)$ & $-0.0001(5)$ & $0.0005(4)$ \\
F5 & $0.0357(7)$ & $0.0126(6)$ & $0.0158(6)$ & $0.0026(5)$ & $-0.0025(5)$ & $0.0015(5)$ \\
F6 & $0.0138(5)$ & $0.0295(7)$ & $0.0145(5)$ & $-0.0016(4)$ & $0.0008(4)$ & $0.0025(5)$ \\
F7 & $0.0183(5)$ & $0.0271(7)$ & $0.0221(6)$ & $0.0067(5)$ & $-0.0018(5)$ & $-0.0056(6)$ \\
F8 & $0.0256(6)$ & $0.0218(6)$ & $0.0139(5)$ & $-0.0114(5)$ & $-0.0010(4)$ & $0.0020(5)$ \\
O1 & $0.0205(6)$ & $0.0135(6)$ & $0.0157(6)$ & $0.0030(5)$ & $0.0022(5)$ & $0.0021(5)$ \\
O2 & $0.0229(6)$ & $0.0139(6)$ & $0.0095(6)$ & $-0.0027(5)$ & $-0.0031(5)$ & $0.0014(5)$ \\
O3 & $0.0181(6)$ & $0.0235(7)$ & $0.0085(5)$ & $-0.0073(6)$ & $-0.0021(4)$ & $0.0004(6)$ \\
O4 & $0.0224(6)$ & $0.0143(6)$ & $0.0097(6)$ & $-0.0018(5)$ & $-0.0029(5)$ & $-0.0011(5)$ \\
C1 & $0.0178(8)$ & $0.0169(8)$ & $0.0116(7)$ & $0.0003(6)$ & $-0.0025(6)$ & $-0.0014(6)$ \\
C2 & $0.0180(7)$ & $0.0147(8)$ & $0.0105(7)$ & $0.0019(7)$ & $-0.0017(6)$ & $0.0021(6)$ \\
C3 & $0.0169(7)$ & $0.0132(8)$ & $0.0111(7)$ & $-0.0024(6)$ & $-0.0010(6)$ & $0.0011(6)$ \\
C4 & $0.0149(7)$ & $0.0134(8)$ & $0.0108(7)$ & $-0.0009(6)$ & $-0.0008(6)$ & $0.0005(6)$ \\
C5 & $0.0149(7)$ & $0.0151(8)$ & $0.0101(7)$ & $-0.0025(6)$ & $0.0004(6)$ & $-0.0007(6)$
\end{tabular}




$\begin{array}{lllllll}\mathrm{C} 6 & 0.0136(6) & 0.0163(8) & 0.0116(7) & -0.0007(7) & -0.0015(5) & -0.0010(6)\end{array}$

Geometric parameters $(\AA, \stackrel{o}{)}$

\begin{tabular}{|c|c|c|c|}
\hline $\mathrm{F} 1-\mathrm{C} 2$ & $1.354(2)$ & $\mathrm{O} 3-\mathrm{C} 4$ & $1.372(2)$ \\
\hline $\mathrm{F} 2-\mathrm{C} 2$ & $1.345(2)$ & $\mathrm{O} 3-\mathrm{C} 5$ & $1.388(2)$ \\
\hline $\mathrm{F} 3-\mathrm{C} 3$ & $1.338(2)$ & $\mathrm{O} 4-\mathrm{C} 6$ & $1.412(2)$ \\
\hline $\mathrm{F} 4-\mathrm{C} 3$ & $1.342(2)$ & $\mathrm{O} 4-\mathrm{H} 4 \mathrm{O}$ & $0.85(4)$ \\
\hline $\mathrm{F} 5-\mathrm{C} 4$ & $1.338(2)$ & $\mathrm{C} 1-\mathrm{C} 2$ & $1.517(3)$ \\
\hline $\mathrm{F} 6-\mathrm{C} 4$ & $1.339(2)$ & $\mathrm{C} 1-\mathrm{H} 1 \mathrm{~A}$ & 0.9900 \\
\hline $\mathrm{F} 7-\mathrm{C} 5$ & $1.354(2)$ & $\mathrm{C} 1-\mathrm{H} 1 \mathrm{~B}$ & 0.9900 \\
\hline $\mathrm{F} 8-\mathrm{C} 5$ & $1.351(2)$ & $\mathrm{C} 3-\mathrm{C} 4$ & $1.547(3)$ \\
\hline $\mathrm{O} 1-\mathrm{C} 1$ & $1.410(2)$ & $\mathrm{C} 5-\mathrm{C} 6$ & $1.513(2)$ \\
\hline $\mathrm{O} 1-\mathrm{H} 1 \mathrm{O}$ & $0.84(4)$ & C6-H6A & 0.9900 \\
\hline $\mathrm{O} 2-\mathrm{C} 3$ & $1.368(2)$ & C6-H6B & 0.9900 \\
\hline $\mathrm{O} 2-\mathrm{C} 2$ & $1.399(2)$ & & \\
\hline $\mathrm{C} 1-\mathrm{O} 1-\mathrm{H} 1 \mathrm{O}$ & $108(2)$ & $\mathrm{F} 4-\mathrm{C} 3-\mathrm{C} 4$ & $108.44(15)$ \\
\hline $\mathrm{C} 3-\mathrm{O} 2-\mathrm{C} 2$ & $120.31(16)$ & $\mathrm{O} 2-\mathrm{C} 3-\mathrm{C} 4$ & $107.76(16)$ \\
\hline $\mathrm{C} 4-\mathrm{O} 3-\mathrm{C} 5$ & $121.70(15)$ & $\mathrm{F} 5-\mathrm{C} 4-\mathrm{F} 6$ & $107.64(16)$ \\
\hline $\mathrm{C} 6-\mathrm{O} 4-\mathrm{H} 4 \mathrm{O}$ & $106(2)$ & $\mathrm{F} 5-\mathrm{C} 4-\mathrm{O} 3$ & $111.28(16)$ \\
\hline $\mathrm{O} 1-\mathrm{C} 1-\mathrm{C} 2$ & $109.99(15)$ & $\mathrm{F} 6-\mathrm{C} 4-\mathrm{O} 3$ & $112.68(16)$ \\
\hline $\mathrm{O} 1-\mathrm{C} 1-\mathrm{H} 1 \mathrm{~A}$ & 109.7 & $\mathrm{~F} 5-\mathrm{C} 4-\mathrm{C} 3$ & $109.64(16)$ \\
\hline $\mathrm{C} 2-\mathrm{C} 1-\mathrm{H} 1 \mathrm{~A}$ & 109.7 & $\mathrm{~F} 6-\mathrm{C} 4-\mathrm{C} 3$ & $109.31(15)$ \\
\hline $\mathrm{O} 1-\mathrm{C} 1-\mathrm{H} 1 \mathrm{~B}$ & 109.7 & $\mathrm{O} 3-\mathrm{C} 4-\mathrm{C} 3$ & $106.26(15)$ \\
\hline $\mathrm{C} 2-\mathrm{C} 1-\mathrm{H} 1 \mathrm{~B}$ & 109.7 & $\mathrm{~F} 8-\mathrm{C} 5-\mathrm{F} 7$ & $105.85(15)$ \\
\hline $\mathrm{H} 1 \mathrm{~A}-\mathrm{C} 1-\mathrm{H} 1 \mathrm{~B}$ & 108.2 & $\mathrm{~F} 8-\mathrm{C} 5-\mathrm{O} 3$ & $111.40(15)$ \\
\hline $\mathrm{F} 2-\mathrm{C} 2-\mathrm{F} 1$ & $106.43(17)$ & $\mathrm{F} 7-\mathrm{C} 5-\mathrm{O} 3$ & $109.52(16)$ \\
\hline $\mathrm{F} 2-\mathrm{C} 2-\mathrm{O} 2$ & $109.02(16)$ & $\mathrm{F} 8-\mathrm{C} 5-\mathrm{C} 6$ & $111.64(16)$ \\
\hline $\mathrm{F} 1-\mathrm{C} 2-\mathrm{O} 2$ & $110.76(15)$ & $\mathrm{F} 7-\mathrm{C} 5-\mathrm{C} 6$ & $111.42(16)$ \\
\hline $\mathrm{F} 2-\mathrm{C} 2-\mathrm{C} 1$ & $110.45(15)$ & $\mathrm{O} 3-\mathrm{C} 5-\mathrm{C} 6$ & $107.05(14)$ \\
\hline $\mathrm{F} 1-\mathrm{C} 2-\mathrm{C} 1$ & $110.80(16)$ & $\mathrm{O} 4-\mathrm{C} 6-\mathrm{C} 5$ & $110.69(14)$ \\
\hline $\mathrm{O} 2-\mathrm{C} 2-\mathrm{C} 1$ & $109.34(16)$ & $\mathrm{O} 4-\mathrm{C} 6-\mathrm{H} 6 \mathrm{~A}$ & 109.5 \\
\hline $\mathrm{F} 3-\mathrm{C} 3-\mathrm{F} 4$ & $107.59(17)$ & $\mathrm{C} 5-\mathrm{C} 6-\mathrm{H} 6 \mathrm{~A}$ & 109.5 \\
\hline $\mathrm{F} 3-\mathrm{C} 3-\mathrm{O} 2$ & $111.10(16)$ & $\mathrm{O} 4-\mathrm{C} 6-\mathrm{H} 6 \mathrm{~B}$ & 109.5 \\
\hline $\mathrm{F} 4-\mathrm{C} 3-\mathrm{O} 2$ & $112.68(16)$ & $\mathrm{C} 5-\mathrm{C} 6-\mathrm{H} 6 \mathrm{~B}$ & 109.5 \\
\hline $\mathrm{F} 3-\mathrm{C} 3-\mathrm{C} 4$ & $109.20(15)$ & $\mathrm{H} 6 \mathrm{~A}-\mathrm{C} 6-\mathrm{H} 6 \mathrm{~B}$ & 108.1 \\
\hline $\mathrm{C} 3-\mathrm{O} 2-\mathrm{C} 2-\mathrm{F} 2$ & $89.9(2)$ & $\mathrm{O} 2-\mathrm{C} 3-\mathrm{C} 4-\mathrm{F} 5$ & $-48.5(2)$ \\
\hline $\mathrm{C} 3-\mathrm{O} 2-\mathrm{C} 2-\mathrm{F} 1$ & $-26.9(2)$ & $\mathrm{F} 3-\mathrm{C} 3-\mathrm{C} 4-\mathrm{F} 6$ & $-169.97(16)$ \\
\hline $\mathrm{C} 3-\mathrm{O} 2-\mathrm{C} 2-\mathrm{C} 1$ & $-149.29(17)$ & $\mathrm{F} 4-\mathrm{C} 3-\mathrm{C} 4-\mathrm{F} 6$ & $-53.01(19)$ \\
\hline $\mathrm{O} 1-\mathrm{C} 1-\mathrm{C} 2-\mathrm{F} 2$ & $-173.74(16)$ & $\mathrm{O} 2-\mathrm{C} 3-\mathrm{C} 4-\mathrm{F} 6$ & $69.23(19)$ \\
\hline $\mathrm{O} 1-\mathrm{C} 1-\mathrm{C} 2-\mathrm{F} 1$ & $-56.1(2)$ & $\mathrm{F} 3-\mathrm{C} 3-\mathrm{C} 4-\mathrm{O} 3$ & $-48.1(2)$ \\
\hline $\mathrm{O} 1-\mathrm{C} 1-\mathrm{C} 2-\mathrm{O} 2$ & $66.3(2)$ & $\mathrm{F} 4-\mathrm{C} 3-\mathrm{C} 4-\mathrm{O} 3$ & $68.84(19)$ \\
\hline $\mathrm{C} 2-\mathrm{O} 2-\mathrm{C} 3-\mathrm{F} 3$ & $77.1(2)$ & $\mathrm{O} 2-\mathrm{C} 3-\mathrm{C} 4-\mathrm{O} 3$ & $-168.91(15)$ \\
\hline $\mathrm{C} 2-\mathrm{O} 2-\mathrm{C} 3-\mathrm{F} 4$ & $-43.8(2)$ & $\mathrm{C} 4-\mathrm{O} 3-\mathrm{C} 5-\mathrm{F} 8$ & $-31.6(2)$ \\
\hline $\mathrm{C} 2-\mathrm{O} 2-\mathrm{C} 3-\mathrm{C} 4$ & $-163.34(16)$ & $\mathrm{C} 4-\mathrm{O} 3-\mathrm{C} 5-\mathrm{F} 7$ & $85.1(2)$ \\
\hline $\mathrm{C} 5-\mathrm{O} 3-\mathrm{C} 4-\mathrm{F} 5$ & $78.4(2)$ & $\mathrm{C} 4-\mathrm{O} 3-\mathrm{C} 5-\mathrm{C} 6$ & $-153.95(17)$ \\
\hline
\end{tabular}




\begin{tabular}{llll}
$\mathrm{C} 5-\mathrm{O} 3-\mathrm{C} 4-\mathrm{F} 6$ & $-42.6(2)$ & $\mathrm{F} 8-\mathrm{C} 5-\mathrm{C} 6-\mathrm{O} 4$ & $59.9(2)$ \\
$\mathrm{C} 5-\mathrm{O} 3-\mathrm{C} 4-\mathrm{C} 3$ & $-162.26(17)$ & $\mathrm{F} 7-\mathrm{C} 5-\mathrm{C} 6-\mathrm{O} 4$ & $-58.2(2)$ \\
$\mathrm{F} 3-\mathrm{C} 3-\mathrm{C} 4-\mathrm{F} 5$ & $72.2(2)$ & $\mathrm{O} 3-\mathrm{C} 5-\mathrm{C} 6-\mathrm{O} 4$ & $-177.92(15)$ \\
$\mathrm{F} 4-\mathrm{C} 3-\mathrm{C} 4-\mathrm{F} 5$ & $-170.79(16)$ & & \\
\hline
\end{tabular}

Hydrogen-bond geometry $\left(A,{ }^{\circ}\right)$

\begin{tabular}{lllll}
\hline$D-\mathrm{H} \cdots A$ & $D-\mathrm{H}$ & $\mathrm{H} \cdots A$ & $D \cdots A$ & $D-\mathrm{H} \cdots A$ \\
\hline $\mathrm{O} 1-\mathrm{H} 1 O \cdots \mathrm{O} 4^{\mathrm{i}}$ & $0.84(4)$ & $1.87(4)$ & $2.705(2)$ & $172(3)$ \\
$\mathrm{O} 4-\mathrm{H} 4 O \cdots \mathrm{O} 1^{\mathrm{ii}}$ & $0.85(4)$ & $1.82(4)$ & $2.661(2)$ & $173(3)$ \\
$\mathrm{C} 6-\mathrm{H} 6 B \cdots \mathrm{F} 4^{\mathrm{iii}}$ & 0.99 & 2.52 & $3.416(2)$ & 151 \\
\hline
\end{tabular}

Symmetry codes: (i) $x, y, z+1$; (ii) $-x+1, y-1 / 2,-z+1$; (iii) $-x+1, y+1 / 2,-z+1$. 